\title{
DERIVATIVES OF MEROMORPHIC FUNCTIONS OF FINITE ORDER
}

\author{
WERNER P. KOHS AND JACK WILLIAMSON
}

\begin{abstract}
Let $F$ be a nonentire, meromorphic function of finite order with only real zeros and real poles such that $F^{\prime}$ has no zeros. We classify all such real $F$ and all such strictly nonreal $F$ whose poles are of bounded multiplicities. We also give examples of such $F$ which are strictly nonreal and whose poles are of unbounded multiplicities.
\end{abstract}

Introduction and statement of the main results. In a series of papers [3, 4, 6] Hellerstein, Shen and Williamson settled an old conjecture of Pólya by characterizing those entire functions $f$ for which $f, f^{\prime}$ and $f^{\prime \prime}$ have only real zeros. The first two of these papers characterized the real entire functions with this property while the third characterized the strictly nonreal entire functions with this property. In this same paper [6], the authors also characterized the strictly nonreal, nonentire meromorphic functions $F$ with only real poles for which $F, F^{\prime}$ and $F^{\prime \prime}$ have only real zeros (a meromorphic function $F$ is real if $z$ real implies $F(z)$ real or infinity; it is strictly nonreal if it is not a constant multiple of a real function).

In this note, we shall prove two theorems related to the problem of characterizing the real, nonentire meromorphic functions $F$ with.only real poles for which $F, F^{\prime}$ and $F^{\prime \prime}$ have only real zeros. We prove

THEOREM 1. Let $F$ be a meromorphic function, not entire, with only real poles. Assume that $F$ and $F^{\prime}$ have only real zeros and that $F^{\prime}$ omits a nonzero value. Then $F$ is of the form

$$
F(z)=A[\tan (a z+b)-B(a z+b)-C],
$$

where $A, B, C, a$ and $b$ are real constants, $a A \neq 0, B \geq 1$, and $C$ has the following property. Let $n$ be an integer such that $-B \pi / 2 \leq C+n B \pi \leq B \pi / 2$. Then, with

$$
\beta=(\sqrt{B-1}+|C+n B \pi|) / B,
$$

we have $\beta<\pi / 2$ and $\tan ^{2} \beta \leq B-1$.

Theorem 1 strengthens a recent result of Hinkkanen and Rossi [8] who proved it under the additional hypotheses that $F$ is real and transcendental. We shall also prove the following theorem.

Received by the editors September 10, 1986 and, in revised form, February 24, 1987.

1980 Mathematics Subject Classification (1985 Revision). Primary 30D30, 30D35.

Key words and phrases. Meromorphic functions, derivative, zeros.

Part of the first author's research towards Ph.D. degree, University of Hawaii. 
THEOREM 2. Let $F$ be a meromorphic function, not entire, of finite order with only real zeros and real poles. Assume that $F^{\prime}$ has no zeros. Then,

(i) if $F$ is real, it is of one of the forms

$$
\begin{gathered}
F(z)=(a z+b)^{-n} \\
F(z)=A \tan (a z+b)+B \\
F(z)=A \frac{a z+b}{c z+d} \\
F(z)=A \frac{(a z+b)^{2}-1}{(a z+b)^{2}}
\end{gathered}
$$

where $A, B, a, b, c$, and $d$ are real constants, $a c A \neq 0$ and where in (2) $n$ is a positive integer, and in (4) $a d \neq b c$,

(ii) if $F$ is strictly nonreal and the multiplicities of its poles are bounded, it is of the form

$$
F(z)=A\left(\frac{e^{-i(b z+c)}}{\sin (b z+c)}\right)^{m}
$$

where $A, b$ and $c$ are constants, $A b \neq 0, b$ and $c$ real, and $m$ is a positive integer.

Theorem 2 strengthens a recent result of Hellerstein, Shen and Williamson [7, Theorem 2] who proved it under the additional hypothesis that the poles of $F$ are simple. Note that if $F$ is nonreal, the zeros of $F^{\prime \prime}$ are real in this case $(m=1)$. However, in the case $m \geq 2, F^{\prime \prime}$ has strictly nonreal zeros, which are given by

$$
z_{k}=\frac{1}{b}\left(\frac{(2 k+1) \pi}{2}-c+\frac{i}{2} \log m\right), \quad k=0, \pm 1, \pm 2, \ldots
$$

REMARK. As will be shown by example in $\S 4$, the hypothesis that the multiplicities of the poles of $F$ are bounded is essential for $F$ to be of the form (6).

1. Preliminaries. The proof of Theorem 2 depends on the following useful fact (see e.g. [3, p. 230 or 11, p. 335]): if $f$ is a real entire function with only real zeros, then

$$
\frac{f^{\prime}}{f}(z)=\phi(z) \psi(z)
$$

where $\phi$ is a real entire function and $\psi$ is a real meromorphic function mapping the upper halfplane into itself. In fact, as a consequence of the proofs of Lemma 4 of [4] and Lemmas 2 and 8 of [3] we have

LEMMA 1. $f$ is of finite order if and only if $\phi$ is a polynomial. In particular $f$ is a Laguerre-Pólya function if $\phi$ is linear.

REMARK. For properties and the Hadamard factorization of functions in the Laguerre-Pólya class (L-P) consult e.g. $[3,4,6]$. A remarkable property of the class of L-P functions is that it is closed under differentiation. This is the only property we will use in the sequel.

We will also need the following lemmas from [7]. 
LEMMA 2. Let $\Pi(z)$ be a canonical product of genus $p$ with only real zeros. Then

$$
\left|\frac{\Pi^{\prime}}{\Pi}(z)\right|=o\left(r^{p}\right)
$$

as $|z|=r \rightarrow \infty, \varepsilon \leq|\arg z| \leq \pi-\varepsilon, \varepsilon>0$.

LEMMA 3. Let $g(z)$ be an entire function of genus $\sigma \geq 2$ with only real zeros. Set $M=[\sigma / 2], \varepsilon=\pi / 2(\sigma+1)$ and let $N$ be any positive integer. Then there are at least $M$ rays re $e^{i \psi_{1}}, \ldots, r e^{i \psi_{M}}$, in $\left\{z=r e^{i \theta}: \varepsilon \leq|\theta| \leq \pi-\varepsilon, 0<r<\infty\right\}$ such that for $k=1, \ldots, M$,

$$
\left|g\left(r e^{i \psi_{k}}\right)\right|=o\left(r^{-N}\right)
$$

as $r \rightarrow \infty$.

LEMMA 4. Let $g(z)$ be a real entire function of genus 0 or 1 with only real zeros. Suppose $g$ has at least $N$ zeros. Then there exists a constant $c>0$ such that

$$
|g(i y)| \geq c|y|^{N}
$$

for $-\infty<y<\infty$.

Lemma 2 is proved by standard growth arguments, while Lemmas 3 and 4 follow from Hadamard's factorization theorem.

2. Proof of Theorem 1. First we will show that $F$ is transcendental. Suppose, on the contrary, that $F$ satisfies the hypotheses of Theorem 1 and that $F=P / Q, P$ and $Q$ polynomials without common zeros. Since $F^{\prime}-\alpha \neq 0$, for some nonzero $\alpha$, we deduce that the polynomial $P^{\prime} Q-P Q^{\prime}-\alpha Q^{2} \neq 0$. By the Fundamental Theorem of Algebra it must be a constant. Differentiation yields $P^{\prime \prime} Q-P Q^{\prime \prime}-2 \alpha Q Q^{\prime} \equiv 0$. Therefore, if $Q\left(z_{0}\right)=0$ for some $z_{0}$, then also $Q^{\prime \prime}\left(z_{0}\right)=0$ with the same multiplicity. This is impossible unless $Q$ is a constant $C$. Hence $P(z)=A z+B$. This shows that $F$ is a polynomial, contradicting the hypothesis that $F$ is not entire.

Next, observe that the equations $F=0, F=\infty$, and $F^{\prime}=\alpha$ have only real zeros. By a result of Edrei [2, Theorem 1] the order of $F$ is at most one. We will now show that $F$ must be real. Indeed, if we assume $F$ is strictly nonreal, then

$$
F^{\prime}(z)=e^{-i(a z+b)} \frac{h(z)}{k(z)},
$$

where $a$ and $b$ are constants, $a \neq 0$ and $h$ and $k$ are real, entire functions of order at most one with only real zeros and without common zeros. On the other hand we can write (since $F^{\prime}$ omits $\alpha$ ),

$$
F^{\prime}(z)=\alpha+\frac{e^{i(c z+d)}}{g(z)}
$$

where $c$ and $d$ are constants and $g$ is a real, entire function of order at most one with only real zeros. Equating (2.1) and (2.2) yields

$$
h(z)-\alpha k(z) e^{i(a z+b)}=e^{i((a+c) z+(b+d))} \frac{k(z)}{g(z)} .
$$


If $h\left(z_{0}\right)-\alpha k\left(z_{0}\right) e^{i\left(a z_{0}+b\right)}=0$ for some $z_{0}$, then $k\left(z_{0}\right)=0$ and thus $h\left(z_{0}\right)=0$. Hence

$$
\begin{gathered}
h(z)-\alpha k(z) e^{i(a z+b)}=C e^{D z}, \\
h^{\prime}(z)-\alpha e^{i(a z+b)}\left(i a k(z)+k^{\prime}(z)\right)=C D e^{D z},
\end{gathered}
$$

where $C$ and $D$ are constants. Now $C \neq 0$, for otherwise $F^{\prime} \equiv \alpha$. Since $k$ has zeros of multiplicity at least two and at least one such zero, there exists a real $x_{0}$ such that $k\left(x_{0}\right)=k^{\prime}\left(x_{0}\right)=0$. By (2.4) and (2.5) we see that $h\left(x_{0}\right)=C e^{D x_{0}}$ and $h^{\prime}\left(x_{0}\right)=C D e^{D x_{0}}$. Since $h\left(x_{0}\right)$ and $h^{\prime}\left(x_{0}\right)$ are real, so are $C$ and $D$. Hence $h(x)-\alpha k(x) e^{i(a x+b)}$ is real for $x$ real. This is equivalent to the assertion that $\alpha e^{i(a x+b)}$ is real for $x$ real. Writing $\alpha=\alpha_{1}+i \alpha_{2}$, we see that

$$
\alpha_{2} \cos (a x+b)+\alpha_{1} \sin (a x+b)=0
$$

for all $x$ real. This last equation can only hold if $\alpha=0$, contradicting the hypothesis that $\alpha$ is not zero. Therefore $a=0$ and $F$ is a transcendental multiple of a real function. Theorem 1 now follows from the result of Hinkkanen and Rossi [8].

3. Proof of Theorem 2. Since $F$ has finite order, we can write

$$
F=e^{P_{1}+i P_{2}} \frac{\Pi_{1}}{\Pi_{2}}
$$

where $P_{1}$ and $P_{2}$ are real polynomials and $\Pi_{1}$ and $\Pi_{2}$ are canonical products of finite genus with only real zeros. Furthermore, since $F^{\prime}$ has no zeros, $\Pi_{1}$ has only simple zeros. Logarithmic differentiation of (3.1) gives

$$
\frac{F^{\prime}}{F}=P_{1}^{\prime}+i P_{2}^{\prime}+\frac{\Pi_{1}^{\prime}}{\Pi_{1}}-\frac{\Pi_{2}^{\prime}}{\Pi_{2}}=\frac{e^{i Q}}{g},
$$

where $Q$ is a real polynomial and $g$ is real entire of finite order with only real zeros.

Now suppose that $F$ is real. Then $P_{2} \equiv 0$ and $Q \equiv 0$, and (3.2) becomes

$$
\frac{F^{\prime}}{F}=P_{1}^{\prime}+\frac{\Pi_{1}^{\prime}}{\Pi_{1}}-\frac{\Pi_{2}^{\prime}}{\Pi_{2}}=\frac{1}{g} .
$$

In view of Lemmas 2 and 3, (3.3) can hold only if $g$ is of genus 0 or 1 . Now set $H=1 / F^{\prime}$. Since $F$ and therefore $F^{\prime}$ is of finite order, $H$ is real, entire of finite order with only real zeros. The logarithmic derivative of $H$ can be written in the form (1.1), so that by (3.3)

$$
\frac{g^{\prime}}{g}-\frac{1}{g}=-\frac{F^{\prime \prime}}{F^{\prime}}=\frac{H^{\prime}}{H}=\phi \psi,
$$

where, by Lemma $1, \phi$ is a polynomial. From (3.4) we get

$$
\phi(i y)=\frac{1}{\psi(i y)}\left(\frac{g^{\prime}}{g}(i y)-\frac{1}{g}(i y)\right) .
$$

Since $\psi$ maps the upper halfplane into itself it satisfies the Carathéodory inequality $[10$, p. 18]

$$
c_{1} \frac{|\sin \theta|}{r}<\left|\psi\left(r e^{i \theta}\right)\right|<c_{2} \frac{r}{|\sin \theta|}
$$


for $r>1$ and $\theta \neq 0, \pi$ and where $c_{1}$ and $c_{2}$ are constants depending on $\psi$ only.

Since $g$ has genus 0 or 1 and $g$ has only real zeros (and at least one since $F$ is not entire), the Hadamard factorization of $g$ and Lemmas 2 and 4 imply

$$
\left|\frac{g^{\prime}}{g}(i y)\right|=o(|y|)
$$

and

$$
|g(i y)| \geq|y|
$$

for $|y|>y_{0}>0$. Equations (3.5)-(3.8) yield

$$
|\phi(i y)|=o\left(|y|^{2}\right) .
$$

This shows that $\phi$ is at most a linear polynomial. Consequently, by Lemma 1 and (3.4), $H$ is an L-P function, and therefore $H^{\prime}$ has only real zeros. Since $F^{\prime}$ has no zeros, (3.4) implies that $F^{\prime \prime}$ has only real zeros. The conclusion now follows from [5, Theorem 3] if $F$ has no zeros and from [7, Theorem 1] if $F$ has at least one zero.

Next, suppose that $F$ is strictly nonreal. Now (3.1) holds with $P_{2}$ nonconstant. Equating imaginary parts of (3.2) implies that for all $z$

$$
P_{2}^{\prime}(z)=\frac{\sin Q(z)}{g(z)}
$$

Thus all but finitely many zeros of $\sin Q(z)$ must be zeros of $g(z)$. Since $g$ has only real zeros, $Q$ is at most linear and since $F$ is strictly nonreal $Q$ is of the form

$$
Q(z)=b z+c
$$

$b, c$ real, $b \neq 0$. Hence (3.2) becomes

$$
P_{1}^{\prime}(z)+i P_{2}^{\prime}(z)+\frac{\Pi_{1}^{\prime}}{\Pi_{1}}(z)-\frac{\Pi_{2}^{\prime}}{\Pi_{2}}(z)=\frac{e^{i(b z+c)}}{g(z)} .
$$

Equating real and imaginary parts of $(3.10)$ gives

$$
\frac{1}{g(z)}=\frac{P_{2}^{\prime}(z)}{\sin (b z+c)}
$$

and

$$
P_{1}^{\prime}(z)+\frac{\Pi_{1}^{\prime}}{\Pi_{1}}(z)-\frac{\Pi_{2}^{\prime}}{\Pi_{2}}(z)=\frac{\cos (b z+c)}{g(z)}=P_{2}^{\prime}(z) \frac{\cos (b z+c)}{\sin (b z+c)} .
$$

Thus by (3.2), (3.9) and (3.11)

$$
\frac{F^{\prime}}{F}(z)=\frac{P_{2}^{\prime}(z)}{\sin (b z+c)} e^{i(b z+c)} .
$$

The following properties are easily deduced.

(P1) By (3.11) the zeros of $P_{2}^{\prime}$ are simple and coincide with zeros of $\sin (b z+c)$.

(P2) By (3.12) and residue considerations, $P_{2}^{\prime}\left(x_{0}\right)=b$ at each zero $x_{0}$ of $\Pi_{1}$ (which is simple and must also be a zero of $\sin (b z+c)$ ). Furthermore, this implies that $\Pi_{1}$ is a polynomial with $\operatorname{deg} \Pi_{1} \leq \operatorname{deg} P_{2}^{\prime}$. To see this, assume $\Pi_{1}$ has more zeros than $\operatorname{deg} P_{2}^{\prime}$. Then $P_{2}^{\prime} \equiv b$, so that by (3.13)

$$
\frac{F^{\prime}}{F}(z)=b \cot (b z+c)+i b
$$


Integration of this formula yields

$$
F(z)=K e^{i(b z+c)} \sin (b z+c),
$$

with $K$ constant, which contradicts the hypothesis that $F$ is not entire.

(P3) By (3.12) and residue considerations, $P_{2}^{\prime}\left(x_{k}\right)=-m_{k} b$ at each zero $x_{k}$ of $\Pi_{2}$ with multiplicity $m_{k}$. Since (P2) and (3.12) imply that the zeros of $\sin (b z+c)$, except for finitely many, are the zeros of $\Pi_{2}$ it follows that $P_{2}^{\prime}$ is of even degree, say $\operatorname{deg} P_{2}^{\prime}=2 d$. This in turn implies that the genus of $\Pi_{2}$ equals $2 d+1$.

To complete the proof, note that from (P3) and the hypothesis that the multiplicities $m_{k}$ are bounded it is easily deduced that $P_{2}^{\prime}=K$, constant. Using (3.13) we obtain

$$
\frac{F^{\prime}}{F}(z)=K \cot (b z+c)+i K
$$

and upon integration

$$
F(z)=A_{1} e^{i K z} \sin ^{K / b}(b z+c) .
$$

By our assumptions, $F$ is meromorphic, not entire. Hence $K=-m b$ for some positive integer $m$. This readily gives

$$
F(z)=A\left(\frac{e^{-i(b z+c)}}{\sin (b z+c)}\right)^{m}
$$

4. An example of a real meromorphic function of finite order with only real zeros and poles, whose poles are of unbounded multiplicities and whose derivative has no zeros. Without loss of generality we may assume that $b=\pi$ and $c=0$ in (3.12) (otherwise a real affine transformation will make it so without affecting the hypotheses of Theorem 2). Then (P1)-(P3) imply that for a suitable constant $a$

$$
P_{2}^{\prime}(z)=a \prod_{i=1}^{2 d}\left(z-\nu_{i}\right), \quad \Pi_{1}(z)=\prod_{j=1}^{p}\left(z-\mu_{j}\right),
$$

where $\nu_{1}<\cdots<\nu_{2 d}, \mu_{1}<\cdots<\mu_{p}, \nu_{i}, \mu_{j}$ integer, $\nu_{i} \neq \mu_{j}(i=1, \ldots, 2 d$, $j=1, \ldots, p)$, and $p \leq 2 d$. Also

$$
P_{2}^{\prime}\left(\mu_{j}\right)=\pi \quad \text { and } \quad P_{2}^{\prime}(k)=-m_{k} \pi
$$

for $j=1, \ldots, p$, where $k$ is an integer, $k \neq \nu_{i}, \mu_{j}(i=1, \ldots, 2 d, j=1, \ldots, p)$; each $m_{k}$ is a positive integer.

Note that, by Rolle's Theorem, (4.1) and (4.2) imply that $\Pi_{1}$ cannot have more than two consecutive zeros.

A complete classification of all polynomials satisfying the equations (4.1) and (4.2) seems to be a very hard problem. Classes of examples can be found in [9].

Let

$$
\Pi_{1}(z)=z, \quad P_{2}^{\prime}(z)=-\pi\left(z^{2}-1\right)
$$

then the multiplicities of the poles of $\Pi_{2}$ in (3.12) are given by

$$
m_{k}=k^{2}-1 \quad(k \neq-1,0,1) .
$$


By property (P3), the genus of the canonical product $\Pi_{2}$ in (3.12) equals three and its zeros are all integers except $-1,0,1$. Hence

$$
\Pi_{2}(z)=\prod_{k \neq-1,0,1}\left[\left(1-\frac{z}{k}\right) \exp \left(\frac{z}{k}+\frac{z^{2}}{2 k^{2}}+\frac{z^{3}}{3 k^{3}}\right)\right]^{k^{2}-1}
$$

and thus

$$
\frac{\Pi_{2}^{\prime}}{\Pi_{2}}(z)=\sum_{k \neq-1,0,1} \frac{z^{3}\left(k^{2}-1\right)}{k^{3}(z-k)} .
$$

By the well-known series expansion of $\cot \pi z$ (see e.g. [1, p. 197])

$$
\cot \pi z=\frac{1}{\pi z}+\frac{1}{\pi} \sum_{k \neq 0} \frac{z}{k(z-k)}
$$

If we now construct the polynomial $P_{1}^{\prime}$ such that (3.12) holds, then $F$, given by (3.1), has the required properties. Solving for $P_{1}^{\prime}$ in equation (3.12) and using (4.3)-(4.6) we see that

$$
\begin{aligned}
P_{1}^{\prime}(z) & =-\frac{1}{z}+\sum_{k \neq-1,0,1} \frac{z^{3}\left(k^{2}-1\right)}{k^{3}(z-k)}-\pi\left(z^{2}-1\right)\left(\frac{1}{\pi z}+\frac{1}{\pi} \sum_{k \neq 0} \frac{z}{k(z-k)}\right) \\
& =\sum_{k \neq-1,0,1} \frac{z\left[z^{2}\left(k^{2}-1\right)-k^{2}\left(z^{2}-1\right)\right]}{k^{3}(z-k)}-3 z \\
& =\sum_{k \neq-1,0,1} \frac{z(k-z)(k+z)}{k^{3}(z-k)}-3 z \\
& =-z^{2} \sum_{k \neq-1,0,1} k^{-3}-z\left(\sum_{k \neq-1,0,1} k^{-2}+3\right) \\
& =-z\left(\frac{\pi^{2}}{3}+1\right) .
\end{aligned}
$$

This gives

$$
P_{1}(z)=-\frac{z^{2}}{2}\left(\frac{\pi^{2}}{3}+1\right)+C
$$

where $C$ is an arbitrary constant.

One can construct more examples of such meromorphic functions $F$ using different pairs of polynomials $P_{2}^{\prime}$ and $\Pi_{1}$, which satisfy (4.1) and (4.2) and by adapting the above technique.

ACKNOWLEDGMENT. The real case of Theorem 2 was suggested by Li-Chien Shen in written communication to Hellerstein and Williamson.

\section{BIBLIOGRAPHY}

1. L. Ahlfors, Complex analysis, McGraw-Hill, 1979.

2. A. Edrei, Meromorphic functions with three radially distributed values, Trans. Amer. Math. Soc. 78 (1955), 276-293. 
3. S. Hellerstein and J. Williamson, Derivatives of entire functions and a question of Pólya, Trans. Amer. Math. Soc. 227 (1977), 227-249.

4. __ Derivatives of entire functions and a question of Pólya. II, Trans. Amer. Math. Soc. 234 (1977), 497-503.

5. _ The zeros of the second derivative of the reciprocal of an entire function, Trans. Amer. Math. Soc. 263 (1981), 501-513.

6. S. Hellerstein, L. C. Shen and J. Williamson, Reality of the zeros of an entire function and its derivatives, Trans. Amer. Math. Soc. 275 (1983), 319-331.

7. __ Real zeros of derivatives of meromorphic functions and solutions of second order differential equations, Trans. Amer. Math. Soc. 285 (1984), 759-776.

8. A. Hinkkanen and J. Rossi, On a problem of Hellerstein, Shen and Williamson, Proc. Amer. Math. Soc. 92 (1984), 72-74.

9. W. P. Kohs, Ph. D. Thesis, University of Hawaii, 1985.

10. B. Ja. Levin, Distribution of zeros of entire functions, Transl. Math. Mono., vol. 5, Amer. Math. Soc., Providence, R. I., 1964.

11. B. Ja. Levin and I. V. Ostrovskii, On the dependence of the growth of an entire function on the distribution of the zeros of its derivatives, Sibirsk. Mat. Zh. 1 (1960), 427-455; English transl., Amer. Math. Soc. Transl. (2) 32 (1963), 323-357.

Department of Mathematics, University of Hawali, Honolulu, hawail 96822 (Current address of Jack Williamson)

Current address (Werner P. Kohs): Pfarrgasse 24, A-7000 Eisenstadt, Austria 